\title{
Do entrapment, injuries, outcomes and potential for self-extrication vary with age? A pre-specified analysis of the UK trauma registry (TARN)
}

\author{
Tim Nutbeam ${ }^{1,2,3^{*}}$ (D) Anthony Kehoe ${ }^{2,4}$, Rob Fenwick ${ }^{5}$, Jason Smith ${ }^{1,6}$, Omar Bouamra ${ }^{7}$, Lee Wallis ${ }^{3}$ and
} Willem Stassen ${ }^{3}$

\begin{abstract}
Background: Motor vehicle collisions (MVCs), particularly those associated with entrapment, are a common cause of major trauma. Current extrication methods are focused on spinal movement minimisation and mitigation, but for many patients self-extrication may be an appropriate alternative. Older drivers and passengers are increasingly injured in MVCs and may be at an increased risk of entrapment and its deleterious effects. The aim of this study is to describe the injuries, trapped status, outcomes, and potential for self-extrication for patients following an MVC across a range of age groups.
\end{abstract}

Methods: This is a retrospective study using the Trauma Audit and Research Network (TARN) database. Patients were included if they were admitted to an English hospital following an MVC from 2012 to 2019. Patients were excluded when their outcomes were not known or if they were secondary transfers. Simple descriptive analysis was used across the age groups: 16-59,60-69, 70-79 and 80+ years. Logistic regression was performed to develop a model with known confounders, considering the odds of death by age group, and examining any interaction between age and trapped status with mortality.

Results: 70,027 patients met the inclusion criteria. Older patients were more likely to be trapped and to die following an MVC ( $p<0.0001)$. Head, abdominal and limb injuries were more common in the young with thoracic and spinal injuries being more common in older patients (all $p<0.0001$ ). No statistical difference was found between the age groups in relation to ability to self-extricate. After adjustment for confounders, the $80+$ age group were more likely to die if they were trapped; adjusted OR trapped 30.2 (19.8-46), not trapped 24.2 (20.1-29.2).

Conclusions: Patients over the age of 80 are more likely to die when trapped following an MVC. Self-extrication should be considered the primary route of egress for patients of all ages unless it is clearly impracticable or unachievable. For those patients who cannot self-extricate, a minimally invasive extrication approach should be employed to minimise entrapment time.

Keywords: Older patients, Extrication, Accidents, Traffic, Spinal injury, Emergency Medical Services

*Correspondence: timnutbeam@nhs.net

1 Emergency Department, University Hospitals Plymouth NHS Trust, Plymouth, UK

Full list of author information is available at the end of the article

\section{Background}

Motor vehicle collisions (MVCs) are a frequent cause of trauma and death for patients of all ages [1]. Following an MVC some patients will be trapped [1]. Only original author(s) and the source, provide a link to the Creative Commons licence, and indicate if changes were made. The images or other third party material in this article are included in the article's Creative Commons licence, unless indicated otherwise in a credit line to the material. If material is not included in the article's Creative Commons licence and your intended use is not permitted by statutory regulation or exceeds the permitted use, you will need to obtain permission directly from the copyright holder. To view a copy of this licence, visit http://creativecommons.org/licenses/by/4.0/. The Creative Commons Public Domain Dedication waiver (http://creativeco mmons.org/publicdomain/zero/1.0/) applies to the data made available in this article, unless otherwise stated in a credit line to the data. 
around $10 \%$ of such patients are physically trapped by deformation of the vehicle, requiring dismantling of the vehicle and space creation by rescue services [2]. The overwhelming majority are trapped due to painful injuries inhibiting movement or physiological impairment rendering voluntary movement difficult [3]. However, often it is rescuer or casualty concerns about exacerbating secondary spinal injury which prevent self or minimally assisted extrication [4, 5].

Being trapped through any of these mechanisms is associated with excess mortality [1]. It is not yet clear whether this can be mitigated by reducing extrication time [6]. Extrication strategies have historically focused on movement mitigation such as roof removal techniques, which inherently take longer to deliver than self-extrication. However, recent work has demonstrated the biomechanical and time advantages of self-extrication over tool-based techniques [7]. Minimising entrapment time reduces avoidable delays to diagnosis and clinical interventions, whilst also reducing the detrimental effects of environmental exposure. A combination of these factors and others may lead to the excess mortality seen in trapped casualties.

Average life expectancy is increasing throughout most of the world, with the most rapidly growing segment of the population being people aged over 60 years [8]. With this changing demographic, healthcare systems have witnessed a disproportionate rise in older patients suffering from major trauma, with this group now representing over $50 \%$ of the major trauma cases reported in the UK [8,9]. There are more older drivers and passengers on the roads than ever before, with older road users representing $12 \%$ of car driving license holders and $9 \%$ of road miles travelled $[10,11]$. Older patients have a higher mortality rate, with those over 70 representing a disproportionate $20 \%$ of all car driver deaths [12].

Older casualties may be at increased risk of entrapment through decreased baseline mobility, a propensity to frailty and vulnerability to certain types of injury [8]. It is unknown if older patients are more at risk from the dangerous effects of prolonged entrapment. Extrication may be delayed due to rescuer perceptions about the incidence of spinal injury in this group and their ability to self-extricate [13].

The aims of this study were to describe the rate of entrapment, the type and frequency of injuries, and outcomes in different age groups, and whether there is disproportionate mortality from entrapment in older patients. We also compared the incidence of factors likely to impede self-extrication between the groups.

\section{Methods}

This is a retrospective review of the UK Trauma Audit and Research Network (TARN) database. TARN is the UK national trauma registry into which all Major Trauma Centres submit data on severely injured patients. TARN moved from voluntary to mandatory submission of data from MVCs in 2012. Eligibility criteria include trauma patients who are admitted to hospital for $\geq 72 \mathrm{~h}$, are admitted to a critical care unit, die in hospital, or are transferred to another hospital for specialist trauma care. Isolated closed fractures of the limbs and hip fractures in patients over 65 are excluded. TARN includes patient demographics, initial physiology, treatment interventions, detail of injuries and in some circumstances (including MVCs) their trapped status.

This study describes the rate of entrapment by age group, considering the effect of being trapped on outcomes and whether this effect modifies with age. Reporting the rate and type of spinal injuries, other severe potentially time critical injuries and traumatic and physiological challenges to self-extrication by age group will inform choice of extrication strategy $[14,15]$.

Patients were included if they were admitted between January 2012 and December 2019, were involved in an MVC, were admitted directly to an English hospital, and had a known outcome. Patients were excluded when their trapped status was not known. For patients who met the inclusion criteria, data fields including age, trapped status, injury severity score (ISS), abbreviated injury score (AIS) for each body region were reported. In addition we report details of spinal injury and other severe injuries that we have previously defined [2].

Adults were categorised into age groups: 16-59, $60-69,70-79$ and $80+$ years. These age groups were selected as they have previously been defined by TARN [8]. The $80+$ age group were considered as a whole to prevent the statistical artifact associated with small sample sizes. Simple descriptive analysis was used to define the characteristics of the groups by age category and trapped status. A two-tailed t-test was used to compare means and Mann-Whitney U test for comparing medians. The Chi square test for uniform distribution was used for categorical variables. $P$ values of less than 0.01 were considered significant due to multiple analyses being performed. Logistic regression was used to develop a model with the following known confounders: sex, ISS, GCS, Charlson comorbidity index and entrapment status as exposure variables, considering the odds of death by age group, and examining any interaction between age and trapped status with mortality. Missing values for GCS were imputed under the assumption of a mechanism of missing at random (MAR). SPSS (IBM Corp v.23 Armonk, NY), 
Stata (StataCorp. 2015. Stata Statistical Software: Release 14. College Station, TX) and R (Integrated Development for R. RStudio, PBC, Boston, MA, v.1.4) software were used for the analyses.

A literature review failed to identify previous studies or guidance which indicates which patients are suitable for self-extrication. All parameters available through the TARN data set were considered by the research group; factors were identified which the group from their clinical and operational experience considered likely to affect the ability of a patient to successfully self-extricate. Factors where consensus was achieved were GCS 12 or less; Spine, Limb or Pelvis Abbreviated Injury Scale (AIS) score of 3+; or a systolic blood pressure of $<90 \mathrm{mmHg}$. Patients where none of these factors were present were considered as having a high potential for self-extrication.

TARN data analyses are conducted using anonymised data which is governed by a code of practice approved by the Confidentiality Advisory Group who are appointed by the Health Research Authority. Additional individual ethical approval was not required for this analysis.

\section{Results}

Between 2012 and 2019 there were 450,437 major trauma cases identified on the TARN database of whom 70,027 met the inclusion criteria (Fig. 1).

The characteristics of each group are summarized in Table 1. The systolic blood pressure increased with age, whilst the pulse, respiratory rate, oxygen saturations and GCS demonstrate statistical though not clinical differences between the groups with no age-dependent trend. With large data-sets there is a well reported tendency for the identification of statistically significant but clinically inconsequential effects [16].

The median ISS was similar across the age groups. Thirty-day mortality increased with increasing age from $4.1 \%(16-59)$ to $16.4 \%(80+)$.

As shown in Table 2, unadjusted and adjusted odds of death increased with age. Trapped patients over 80 had an increased mortality rate compared to those that were not trapped (Fig. 2). This model performed well, with a discrimination area under the receiver operator curve (ROC, C-statistic) of 0.952 (95\% CI 0.948-0.955) as shown in Fig. 3.

In patients who were trapped, severe injuries occurred with similar frequency across all age categories (Table 3).

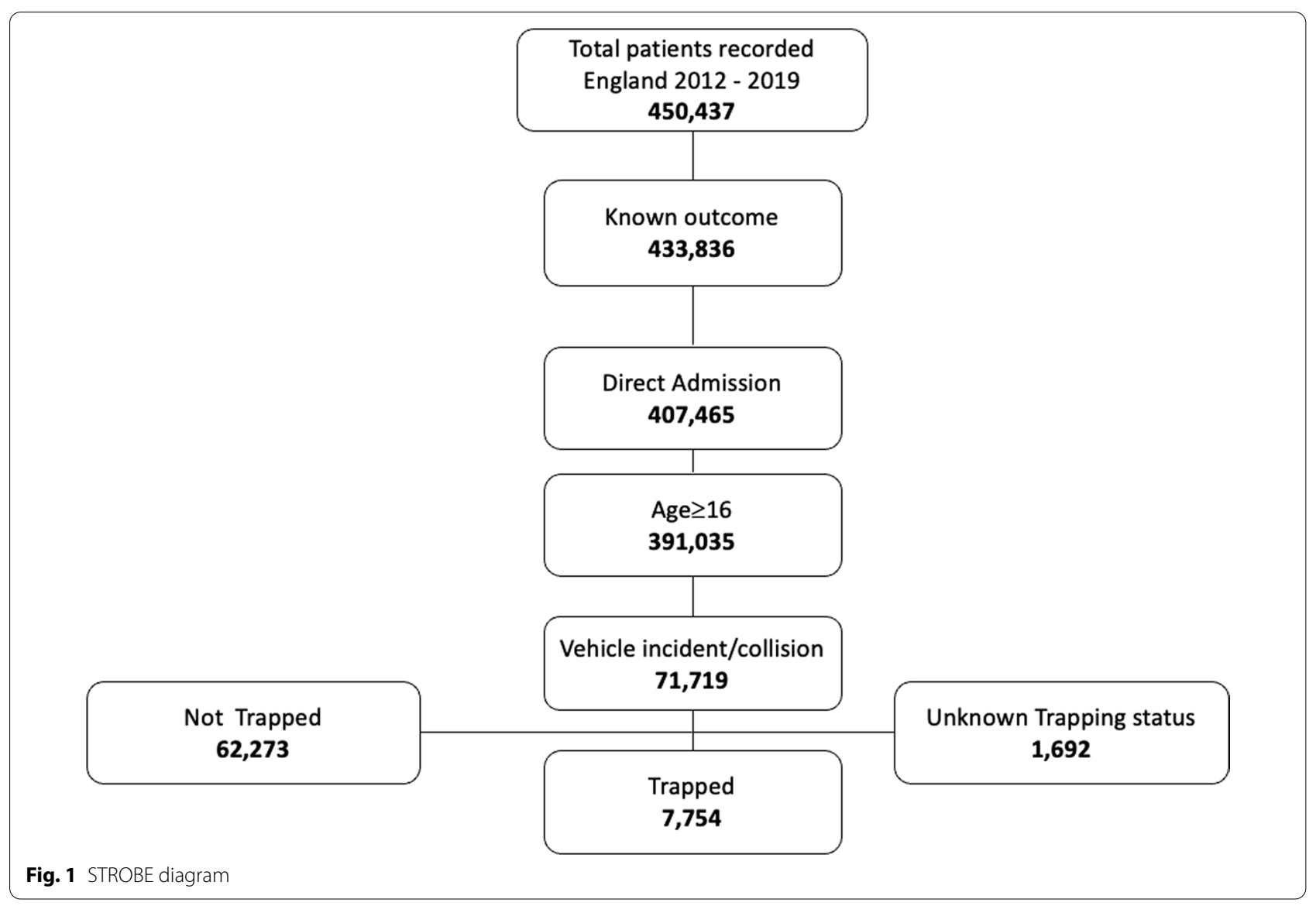


Table 1 Demographics and mortality by age

\begin{tabular}{|c|c|c|c|c|c|}
\hline Age groups & Total & $16-59$ & $60-69$ & $70-79$ & $80+$ \\
\hline Total number of cases & 70,027 & 51,868 & 7605 & 5733 & 4821 \\
\hline Male, n (\%) & $51,852(74 \%)$ & $40,957(79 \%)$ & $5232(68.8 \%)$ & $3197(55.8 \%)$ & $2466(51.2 \%)$ \\
\hline ISS, median (IQR) & $13(9-22)$ & $13(9-24)$ & $13(9-22)$ & $13(9-24)$ & $13(9-22)$ \\
\hline Systolic BP, mean (SD) & $133(28)$ & $129(25)$ & $140(30)$ & $145(33)$ & $149(34)$ \\
\hline Pulse rate, mean (SD) & $87(22)$ & $88(22)$ & $83(21)$ & $83(22)$ & $83(21)$ \\
\hline Respiratory rate, mean (SD) & $20(7)$ & $20(7)$ & $20(7)$ & $20(7)$ & $20(7)$ \\
\hline Oxygen saturation, mean (SD) & $96(8)$ & $96(8)$ & $96(8)$ & $95(9)$ & $95(7)$ \\
\hline GCS, median (IQR) & $15(15-15)$ & $15(15-15)$ & $15(15-15)$ & $15(15-15)$ & $15(15-15)$ \\
\hline Trapped, n (\%) & $7754(11.1 \%)$ & $5642(10.9 \%)$ & $807(10.6 \%)$ & $756(13.2 \%)$ & $549(11.4 \%)$ \\
\hline Mortality, n (\%) & $3868(5.5 \%)$ & $2125(4.1 \%)$ & $391(5.1 \%)$ & $564(9.8 \%)$ & $788(16.4 \%)$ \\
\hline
\end{tabular}

IQR interquartile range, SD standard deviation

Statistically significant differences $(p<0.0001)$ were found across all groups apart from in Respiratory rate and GCS catergories

Table 2 Trapped status and mortality by age

\begin{tabular}{|c|c|c|c|c|c|c|c|c|}
\hline \multirow[t]{2}{*}{ Age groups } & \multicolumn{4}{|c|}{ Trapped at scene } & \multicolumn{4}{|c|}{ Not trapped at scene } \\
\hline & $16-59$ & $60-69$ & $70-79$ & $80+$ & $16-59$ & $60-69$ & $70-79$ & $80+$ \\
\hline $\begin{array}{l}\text { Unadjusted odds } \\
\text { ratio of death } \\
(95 \% \mathrm{Cl})\end{array}$ & 1 & $1.1(0.9-1.5)$ & $1.7(1.4-2.2)$ & $4.4(3.6-5.5)$ & 1 & $1.3(1.2-1.5)$ & $2.7(2.5-3.0)$ & $4.6(4.2-5.1)$ \\
\hline $\begin{array}{l}\text { Adjusted odds } \\
\text { ratio of death } \\
(95 \% \mathrm{Cl})\end{array}$ & 1 & $3.7(2.3-5.9)$ & $8.5(5.5-13.3)$ & $30.2(19.8-46)$ & 1 & $2.8(2.3-3.4)$ & $8.7(7.2-10.6)$ & $24.2(20.1-29.2)$ \\
\hline
\end{tabular}

Adjusted for gender, ISS, GCS, Comorbidity

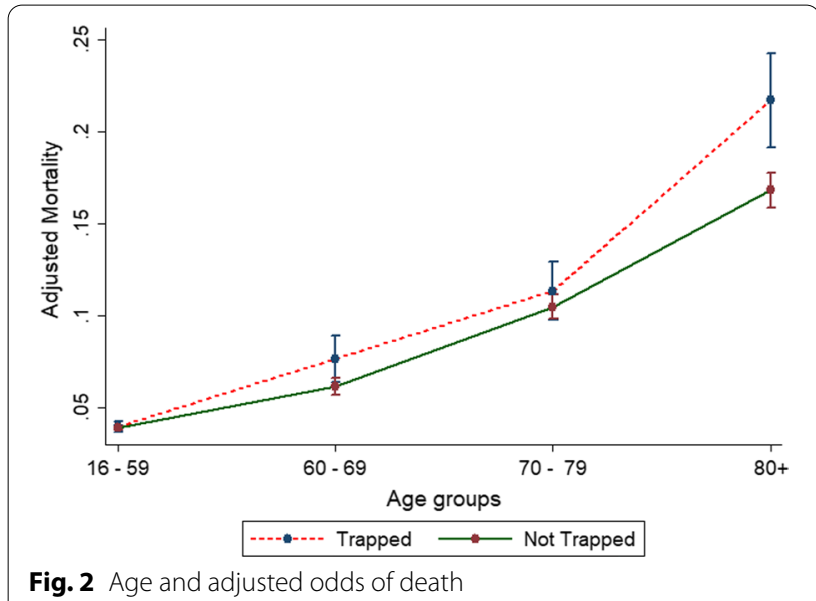

Injuries (AIS $3+$ ) to the head, face, abdomen and limbs were more common in the young (16-59, Table 4). Thoracic injuries were more frequent in those aged 60 or above.

The frequency of multiple spinal fractures, dens fractures, unstable fractures and cord injuries were highest in the 70-79 age group.

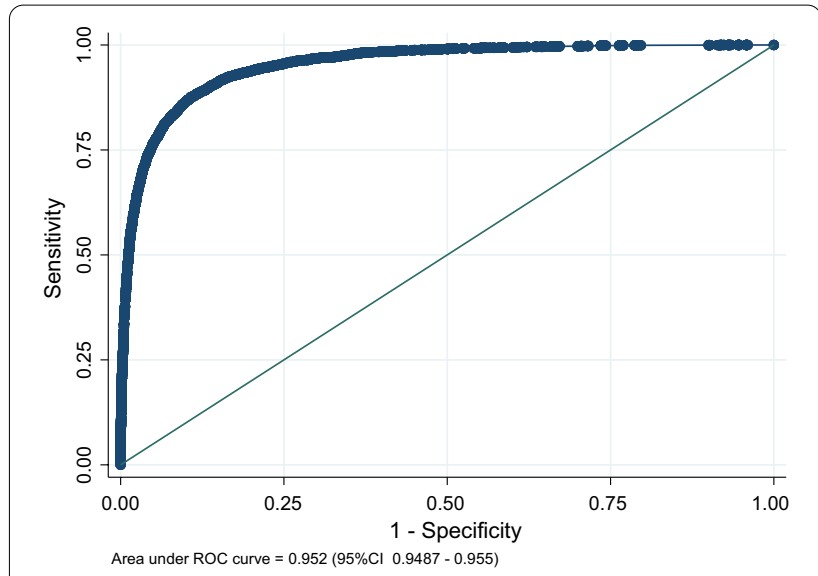

Fig. 3 Receiver operator curve for model ( ${ }^{*}$ Gender, ISS, GCS, Charlson comorbidity index and entrapment status as exposure variables)

Statistically significant but not clinically significant differences were demonstrated across the physiological and injury-based considerations for self-extrication. The proportion of patients with injuries likely to preclude self-extrication was similar across the age groups (Table 5). 
Table 3 Severe and spinal injuries by age for trapped casualties

\begin{tabular}{|c|c|c|c|c|c|c|}
\hline \multirow[t]{2}{*}{ Injury } & \multirow[t]{2}{*}{ Total } & \multicolumn{4}{|l|}{ Age group } & \multirow[t]{2}{*}{ Significance $(p)$} \\
\hline & & $16-59$ & $60-69$ & $70-79$ & $80+$ & \\
\hline Pelvic Ring Blood loss $>20 \%$ & $71(0.9 \%)$ & $53(0.9 \%)$ & $9(1.1 \%)$ & $5(0.7 \%)$ & $4(0.7 \%)$ & 0.7578 \\
\hline Blood Loss > 20\% & $275(3.5 \%)$ & $210(3.7 \%)$ & $28(3.5 \%)$ & $20(2.6 \%)$ & $17(3.1 \%)$ & 0.4503 \\
\hline Tension pneumothorax & $118(1.5 \%)$ & $91(1.6 \%)$ & $12(1.5 \%)$ & $8(1.1 \%)$ & $7(1.3 \%)$ & 0.6535 \\
\hline Spine multiple fractures & $1078(13.9 \%)$ & $734(13 \%)$ & $114(14.1 \%)$ & $150(19.8 \%)$ & $80(14.6 \%)$ & $<0.0001$ \\
\hline Spine dens fracture & $164(2.1 \%)$ & $87(1.5 \%)$ & $19(2.4 \%)$ & $37(4.9 \%)$ & $21(3.8 \%)$ & $<0.0001$ \\
\hline Spine compression grade 2 and 3 & $141(1.8 \%)$ & $98(1.7 \%)$ & $13(1.6 \%)$ & $17(2.2 \%)$ & $13(2.4 \%)$ & 0.5485 \\
\hline Spine unstable fracture & $717(9.2 \%)$ & $502(8.9 \%)$ & $79(9.8 \%)$ & $94(12.4 \%)$ & $42(7.7 \%)$ & 0.0077 \\
\hline Spinal cord injury & $526(6.8 \%)$ & $376(6.7 \%)$ & $51(6.3 \%)$ & 63 (8.3\%) & 36 (6.6\%) & 0.3452 \\
\hline
\end{tabular}

Table 4 Injury site (AIS 3+) by age for trapped casualties

\begin{tabular}{|c|c|c|c|c|c|c|}
\hline \multirow[t]{2}{*}{ Anatomical site } & \multirow[t]{2}{*}{ Total } & \multicolumn{4}{|l|}{ Age group } & \multirow[t]{2}{*}{ Significance $(p)$} \\
\hline & & $16-59$ & $60-69$ & $70-79$ & $80+$ & \\
\hline Head & $1896(24.5 \%)$ & $1528(27.1 \%)$ & 135 (16.7\%) & 139 (18.4\%) & 94 (17.1\%) & $<0.0001$ \\
\hline Face & $52(0.7 \%)$ & $43(0.8 \%)$ & $3(0.4 \%)$ & $5(0.7 \%)$ & $1(0.2 \%)$ & $<0.0001$ \\
\hline Thorax & $4159(53.6 \%)$ & 2945 (52.2\%) & $438(54.3 \%)$ & 430 (56.9\%) & 346 (63\%) & $<0.0001$ \\
\hline Abdomen & $950(12.3 \%)$ & 770 (13.6\%) & $74(9.2 \%)$ & $65(8.6 \%)$ & $41(7.5 \%)$ & $<0.0001$ \\
\hline Spine & 844 (10.9\%) & $577(10.2 \%)$ & $96(11.9 \%)$ & 109 (14.4\%) & $62(11.3 \%)$ & $<0.0001$ \\
\hline Pelvic & 895 (11.5\%) & $686(12.2 \%)$ & $82(10.2 \%)$ & $60(7.9 \%)$ & $67(12.2 \%)$ & $<0.0001$ \\
\hline Limb & $2522(32.5 \%)$ & 2028 (35.9\%) & $232(28.7 \%)$ & 164 (21.7\%) & $98(17.9 \%)$ & $<0.0001$ \\
\hline
\end{tabular}

Table 5 Physiological and injury considerations for potential for self-extrication by age

\begin{tabular}{|c|c|c|c|c|c|c|}
\hline \multirow[t]{2}{*}{ Parameter } & \multirow[t]{2}{*}{ Total } & \multicolumn{4}{|l|}{ Age group } & \multirow[t]{2}{*}{ Significance $(p)$} \\
\hline & & $16-59$ & $60-69$ & $70-79$ & $80+$ & \\
\hline Systolic BP $<90$ & $418(5.4 \%)$ & 301 (5.3\%) & $48(6.0 \%)$ & $39(5.2 \%)$ & $30(5.5 \%)$ & 0.908 \\
\hline GCS 12 or less & 1183 (15.3\%) & 1006 (17.8\%) & $68(8.4 \%)$ & $57(7.5 \%)$ & $52(9.5 \%)$ & $<0.0001$ \\
\hline Spine AIS3+ & 844 (10.9\%) & $577(10.2 \%)$ & $96(11.9 \%)$ & 109 (14.4\%) & $62(11.3 \%)$ & $<0.0001$ \\
\hline Pelvic AlS 3+ & 895 (11.5\%) & $686(12.2 \%)$ & $82(10.2 \%)$ & $60(7.9 \%)$ & $67(12.2 \%)$ & $<0.0001$ \\
\hline Limb AIS 3+ & $2522(32.5 \%)$ & $2028(35.9 \%)$ & $232(28.7 \%)$ & $164(21.7 \%)$ & $98(17.9 \%)$ & $<0.0001$ \\
\hline None of the above & 3208 (41.4\%) & $2264(40.1 \%)$ & $343(42.5 \%)$ & $357(47.2 \%)$ & $244(44.4 \%)$ & 0.079 \\
\hline
\end{tabular}

\section{Discussion}

Patients over 80 years old are particularly vulnerable to the negative effects of entrapment following an MVC. Older patients are more likely to have chest and spinal injuries than younger patients-however, the overall rate of spinal injuries in comparison to other likely time dependent injuries remains low. Across the age groups, approximately $40 \%$ of patients who were trapped did not have injuries or physiological impairment likely to hinder self-extrication.

\section{Meaning of the study}

This study offers fresh insights that are useful for those providing clinical care on scene, planning extrication strategies and supporting clinicians in enabling patients to self-extricate. Injuries of the head, thorax, face and limb are unlikely to benefit from a longer extrication strategy based on movement mitigation when other quicker routes such as self-extrication could be considered; these injuries may be time dependent and the extended time these patients remain in the vehicle will 
add to excess mortality related to bleeding and hypoxia $[10,17]$. Gentle patient handling and movement mitigation may help with prevention of clot disruption in abdominal or pelvic injury [18], but these significant injuries often require blood product resuscitation and early access to hospital-based services for identification of injury (CT scan) and treatment (interventional radiology or damage control surgery) [19].

The small increased rate of spinal injuries in older patients may be because of the decreased bone density, muscle and ligament strength and degenerative changes causing narrowing of the spinal canal experienced by the older patient [13]. Recent work has identified that self-extrication results in less movement of the cervical and lumbar spine than other extrication types in healthy volunteers [7]. If these findings can be extrapolated to the injured population, self-extrication may present the best route of egress even for those with suspected spinal injuries.

Patients, and particularly older patients may have occult injuries [20]. As such, predicting a patient's ability to self-extricate is complex. We suggest that selfextrication has significant advantages over more formal alternative extrication techniques and as such should be considered as a route of egress for all patients unless it is clearly impracticable or unachievable. The advantages of self-extrication for the patient include minimal entrapment time (self-extrication is quickest) and minimal movement [7]. For those patients who cannot self-extricate a minimally invasive extrication approach should be used-providing the patient with the necessary support to extricate from the vehicle with minimal cutting/space creation using the principles of gentle patient handling.

\section{Strengths and weaknesses}

This is the largest analysis to date of trapped patients injured in motor vehicle collisions, which allows comparison of injury severity, injury type and outcomes for patients stratified by age.

TARN data may be incomplete, with patients aged over 60 having a lower level of data completeness than younger patients [8]. This study is based upon chronological age-the effects of which are subject to considerable variation between individuals [21]. This study does not specifically report frailty-which is likely to be an important factor both in a patient's resilience to injury and their potential to recover successfully from injury and therefore affect both injury severity and mortality [22].

We have selected pragmatic physiological and injurybased criteria which are likely to affect the ability of a patient to participate in self-extrication. These criteria have not been validated in this setting but provide useful context.

This study is limited in that it does not report nonpatient factors relating to the scene of a collision which will affect clinical decision making. We do not report type of vehicle, closing speed, vehicle damage or the use and/or deployment of restraint systems. Importantly we cannot distinguish between patients that are physically trapped and those that are medically trapped following their MVC.

\section{Unanswered questions and future research}

Future work should focus on clearly defining patient groups that are not suitable for self-extrication. This may be through prospective data collection of extrication type and patient outcomes, expert consensus, and patient consultation. It is important to distinguish between patients who are physically trapped and those that are medically trapped, and this should be routinely collected on operational and medical data sets considering trapped patients post MVC.

\section{Conclusions}

Patients over the age of 80 are more likely to die when trapped following an MVC. Older patients are more likely to have chest and spinal injuries than younger patients-however, the overall rate of spinal injuries remains low across all age groups. Older patients are no more likely to have injuries that would hinder selfextrication than younger patients.

Self-extrication should be considered the primary route of egress for patients of all ages apart from where it is clearly impracticable or unachievable. For those patients who cannot self-extricate a minimally invasive extrication approach should be employed to minimise entrapment time.

\section{Acknowledgements \\ We would like to thank TARN and the TARN research committee for their support.}

\section{Authors' contributions}

$\mathrm{TN}, \mathrm{AK}, \mathrm{RF}, \mathrm{JS}, \mathrm{LW}$ and WS contributed to the conception and study design, analysis and interpretation of data, drafting and revising the manuscript. $O B$ contributed to the analysis and interpretation of the data and critically revised the manuscript. All authors read and approved the final manuscript.

\section{Funding}

Publication costs were funded by a charitable grant from the Road Safety Trust. The Road Safety Trust had no role in the design, data collection, analysis or writing of this manuscript.

\section{Availability of data and materials}

The datasets used and/or analysed during the current study are available from the corresponding author on reasonable request. 


\section{Declarations}

\section{Ethics approval and consent to participate}

TARN data analyses are conducted using anonymised data which is governed by a code of practice approved by the Confidentiality Advisory Group who are appointed by the Health Research Authority. Additional individual ethical approval was not required for this analysis.

\section{Consent for publication}

Not applicable.

\section{Competing interests}

The authors declare that they have no competing interests.

\section{Author details}

${ }^{1}$ Emergency Department, University Hospitals Plymouth NHS Trust, Plymouth, UK. ${ }^{2}$ Devon Air Ambulance Trust, Exeter, UK. ${ }^{3}$ Division of Emergency Medicine, University of Cape Town, Cape Town, South Africa. ${ }^{4}$ Emergency Department, Somerset NHS Foundation Trust, Taunton, UK. ${ }^{5}$ University Hospitals Birmingham, Birmingham, UK. ${ }^{6}$ Academic Department of Military Emergency Medicine, Royal Centre for Defence Medicine, Birmingham, UK. ${ }^{7}$ Trauma Audit Research Network, University of Manchester, Manchester, UK.

Received: 15 November 2021 Accepted: 26 December 2021

Published online: 05 March 2022

\section{References}

1. Nutbeam T, Fenwick R, Smith J, Bouamra O, Wallis L, Stassen W. A comparison of the demographics, injury patterns and outcome data for patients injured in motor vehicle collisions who are trapped compared to those patients who are not trapped. Scand J Trauma Resusc Emerg Med. 2021;29:17.

2. Fenwick R, Nutbeam T. Medical vs. true physical traffic collision entrapment. J Paramed Pract. 2018;10:158-62.

3. Fenwick R, Nutbeam T, Dayson M. A 10-year time series analysis of roof removal extrications by Fire and Rescue Services in England. In: Faculty of Prehospital Care Scientific Conference; 2020.

4. NDFFAEM. Road Traffic Accident Handbook; 2009.

5. Brown A, Low A. Self-extrication and selective spinal immobilisation in a polytrauma patient with spinal injuries. Trauma. 2020;22:229-32.

6. Nutbeam T, Fenwick R, Hobson C, Holland V, Palmer M. Extrication time prediction tool. Emerg Med J. 2015;32:401.

7. Nutbeam T, Fenwick R, May B, et al. Assessing spinal movement during four extrication methods: a biomechanical study using healthy volunteers. Scand J Trauma Resusc Emerg Med. 2022;30:7. https://doi.org/10. 1186/s13049-022-00996-5.

8. TARN. Major Trauma in Older People. 2017; Available from: https://www. tarn.ac.uk/content/downloads/3793/Major\%20Trauma\%20in\%200lder\% 20People\%202017.pdf.

9. Kehoe A, Smith JE, Edwards A, Yates D, Lecky F. The changing face of major trauma in the UK. Emerg Med J. 2015;32:911-5.

10. Deynse HV, Belleghem GV, Hubloue I, Lauwaert D, Moens M, Pien K, et al. The incremental hospitalization cost of significant transport-related traumatic brain injury. J Head Trauma Rehab. 2020;35:E144-55.

11. Abou-Raya S, ElMeguid LA. Road traffic accidents and the elderly. Geriatr Gerontol Int. 2009;9:290-7.

12. A P. Supporting safe driving into old age: a national older driver strategy; 2016.

13. Ikpeze TC, Mesfin A. Spinal cord injury in the geriatric population: risk factors, treatment options, and long-term management. Geriatric Orthop Surg Rehabil. 2017:8:115-8.

14. Dunbar I. Vehicle extrication - the next generation. LUKAS; 2021.

15. NFCC. National Operational Guidance [Internet]. National Operational Guidance. 2021 [cited 2021 Oct 21]. Available from: https://www.ukfrs. com/guidance/.

16. Kaplan RM, Chambers DA, Glasgow RE. Big data and large sample size: a cautionary note on the potential for bias. Clin Transl Sci. 2014;7:342-6.
17. Azami-Aghdash S, Aghaei MH, Sadeghi-Bazarghani H. Epidemiology of road traffic injuries among elderly people; a systematic review and metaanalysis. Bull Emerg Trauma. 2018;6:279-91.

18. Scott I, Porter K, Laird C, Greaves I, Bloch M. The prehospital management of pelvic fractures: initial consensus statement. Emerg Med J. 2013;30:1070

19. Midwinter MJ, Woolley T. Resuscitation and coagulation in the severely injured trauma patient. Philos Trans R Soc B Biol Sci. 2011;366:192-203.

20. Hatton GE, McNutt MK, Cotton BA, Hudson JA, Wade CE, Kao LS. Agedependent association of occult hypoperfusion and outcomes in trauma. J Am Coll Surg. 2020;230:417-25.

21. Brayne C, Dufouil C, Ahmed A, Dening TR, Chi L-Y, McGee M, et al. Very old drivers: findings from a population cohort of people aged 84 and over. Int J Epidemiol. 2000;29:704-7.

22. Bond EG, Durbin LL, Cisewski JA, Qian M, Guralnik JM, Kasper JD, et al. Association between baseline frailty and driving status over time: a secondary analysis of the national health and aging trends study. Inj Epidemiol. 2017:4:9.

\section{Publisher's Note}

Springer Nature remains neutral with regard to jurisdictional claims in published maps and institutional affiliations.

Ready to submit your research? Choose BMC and benefit from

- fast, convenient online submission

- thorough peer review by experienced researchers in your field

- rapid publication on acceptance

- support for research data, including large and complex data types

- gold Open Access which fosters wider collaboration and increased citations

- maximum visibility for your research: over 100M website views per year

At BMC, research is always in progress.

Learn more biomedcentral.com/submissions 